\title{
Towards Autonomous Surveying of Underground Mine using MAVs *
}

\author{
Christoforos Kanellakis, Sina Sharif Mansouri, George Georgoulas, and George \\ Nikolakopoulos \\ Robotics Group, Department of Computer, Electrical and Space Engineering, Luleå \\ University of Technology, Luleå SE-97187, Sweden, Emails: \\ \{chrkan, sinsha, geogeo, geonik\}@ltu.se
}

\begin{abstract}
Micro Aerial Vehicles (MAVs) are platforms that received great attention during the last decade. Recently, the mining industry has been considering the usage of aerial autonomous platforms in their processes. This article initially investigates potential application scenarios for this technology in mining. Moreover, one of the main tasks refer to surveillance and maintenance of infrastructure assets. Employing these robots for underground surveillance processes of areas like shafts, tunnels or large voids after blasting, requires among others the development of elaborate navigation modules. This paper proposes a method to assist the navigation capabilities of MAVs in challenging mine environments, like tunnels and vertical shafts. The proposed method considers the use of Potential Fields method, tailored to implement a sense-and-avoid system using a minimal ultrasound-based sensory system. Simulation results demonstrate the effectiveness of the proposed strategy.
\end{abstract}

Keywords: MAV, Underground Mines, Navigation

\section{Introduction}

MAVs are agile platforms that have emerged over the past decade. These platforms have the ability to fly in different modes, aggressively, smoothly, hover close to a target and perform advanced maneuvers. The mining industry has recently shown interest in the incorporation of MAVs in their process, as an assistance tool to boost production and secure a safer working place in underground challenging conditions. The potential benefits of deploying aerial platforms underground include access to unreachable and dangerous locations and monitoring personnel in unsafe areas. These attributes will have imminent impact on the mine operation, production and safety. So far there have been developed multiple systems, limited to remotely operate in open pit mines above ground and assist in stockpile surveying, 3D pit model build, facility monitoring, security inspection and environmental assessment of the mine sites. The effort to introduce

*This work has been partially funded by the European Unions Horizon 2020 Research and Innovation Programme under the Grant Agreement No.730302 - SIMS. 
aerial robotic platform underground, needs to address multiple challenges before reaching an increased technology readiness level for semi/full autonomous operation. Briefly, harsh underground environment pose obstacles for flying MAVs, like the narrow passages, reduced visibility due to rock falls, dust, wind gusts and lack of proper illumination, all of which constitute necessary the development of elaborate control, navigation, and perception modules for these vehicles.

Within the related literature of MAVs in underground mine operations, few research efforts have been reported trying to address these challenging tasks. In [I] a visual inertial navigation framework has been proposed, to implement position tracking control of the platform. In this case, the MAV was controlled to follow obstacle free paths, while the system was experimentally evaluated in real scale in a tunnel environment, simulating a coal mine, were the illumination challenge was assumed solved. In [2] a more realistic approach, compared to [I] regarding underground localization, has been performed. More specifically, a hexacopter equipped with a Visual Inertial sensor and a laser scanner was manually guided across a vertical mine shaft to collect data for post-processing. The extracted information from the measurements have been utilized to create a 3D mesh of the environment and localize the vehicle. Finally, in [3] the estimation, navigation, mapping and control capabilities for autonomous inspection of penstocks and tunnels using aerial vehicles has been studied, using IMUs, cameras and lidar sensors.

Inspired by the vision of underground aerial robotic platforms, this paper tries to push the current state of the art a step further and contribute in the related field. More specifically, the article provides a detailed and thorough discussion on the potential application scenarios for MAVs in underground mines, identifying the major challenges that need to be addressed prior to actual experimentation. This discussion considers the development of a knowledge basis on this field, to provide an insight on the required mine tasks driven by the mining companies needs, as an enabler for further research directions towards the vision of MAVs in mines. Additionally, as mentioned above, autonomous aerial platforms apart from sensing capabilities rely also in advanced navigation algorithms [4]. This work focused on the development of a path planning scheme to enhance the obstacle avoidance capabilities of multirotors while flying in a tunnel or vertical shafts. It proposes an efficient navigation sense-and-avoid system based on ultrasound sensory system, to securely drive the multirotors around in challenging mine areas, avoiding obstacles in their way. The proposed local trajectory re-planner is based on Potential Fields, applied for the case of $3 \mathrm{D}$ navigation in such a way as to avoid local minima. The implementation of the discussed method requires inexpensive computational sources, increasing the applicability to any platform with adequate computational and sensing capabilities on-board. Finally, multiple simulation results demonstrate the performance of the method, using the open source simulation framework Gazebo that implements realistic flight dynamics.

The rest of this paper is organized as follows. In Section $\square$ the potential application scenarios are presented, while in Section [3 the proposed obstacle-free 


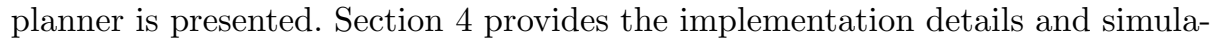
tion results. Finally Section 1 includes the concluding remarks of the paper.

\section{Application Scenarios for MAVs in Underground Mines}

There is an emerging effort from mining companies, currently, to test, evaluate and define possible application scenarios for MAVs towards the future mine. This process requires the combination of the multirotor capabilities with the mine operation needs. Within this work some scenarios are defined for flying vehicles. More specifically, the main objectives for the autonomous/semi-autonomous robotic platforms for surveillance activities include:

- Inspection of shafts for determining their status, where the full suite of sensor can be utilized for generating 3D maps and differences in an area over the entire length of the shaft, while the onboard control systems will assist the pilot by collision avoidance, or will put the system in full autonomous mode for automatic inspections.

- Inspection of hazardous areas while also generating maps or other valuable metrics that the onboard sensor systems can measure, such as gas sensors, dust sensors, and video streams to name a few.

- Inspection of large voids after blasting, where visual navigation systems together with other sensors can be used to either map out the void or provide valuable metrics, such as volume of the void or proximity to other active mining areas or personnel.

The application of MAVs in mining inspection is one of the most enabling factors towards the vision of Zero Production Areas (ZEPA) [5]. As it has been massively investigated lately there is a huge potential for this technology to create a safer working environment in the mines, while at the same time increase the production and reduce the overall operating cost as well as the response of the machines to ad-hoc defined scenarios (always based on the productions needs).

\subsection{Challenges}

The main challenge within this scenario is the mine environment itself, mainly due the possible narrow operational areas, the uneven surfaces and the dusty environments. Since narrow areas and uneven surfaces can be addressed by software development, the biggest challenge would be the dust clouds covering sensors and filling in the mechanical components of the MAV. Figure $\mathbb{U}$ depicts the mine typical underground tunnel. The major identified challenges when operating MAVs underground are listed below:

Wind Gusts: The MAV will operate in an environment with sudden and relatively powerful wind gusts. Wind gusts can occur both in ventilation shafts as well as in ore passes. The wind gusts have a significant effect on the online execution of the path following and thus the better the control scheme is, the better the path following will be. 


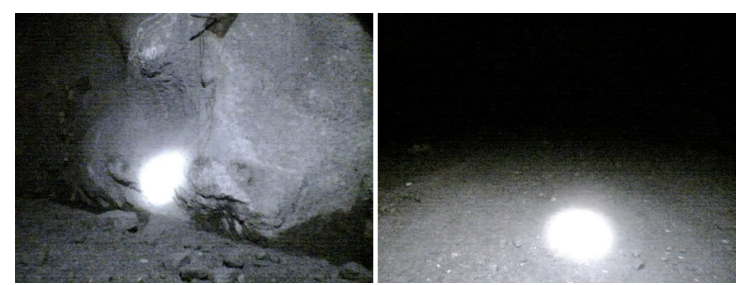

Fig. 1: Snapshots of a typical mine environment.

Dust: Dust is commonly found in iron ore mine areas and in general pose serious problems, especially when it can be produced by the downwash from the propellers. Dust blocks the camera field of view deteriorating its performance causing inaccurate results. Additionally magnetic dust can damage the electronic hardware and therefore specific solutions to shield and protect the hardware parts are essential for long term operations.

Limited Flight Time Generally, the problem of limited flight time [6] for a MAV can be addressed with various strategies that fulfill the task requirements. Firstly, multiple MAV can be employed to share responsibilities and areas of coverage in a way to cooperatively explore and inspect areas like shafts, large voids and tunnels decreasing the inspection time.

Illumination: Visual sensors require a substantial amount of light in the environment in order to provide accurate results. Thereafter, specific lightening strategies should be followed to guarantee proper ambient lighting without affecting the way cameras perceive the environment.

Narrow passages: The diameter of the ore pass can narrow down in extreme cases. The challenge in this case is when such a passage is far away from the position of the pilot. Moreover the collision avoidance system needs to be robust to: 1) loss of sensors, 2) dust gathered on the sensors, and 3) sensor outliers.

Path planning: When the operator does not have line of sight to the MAV, or the mission requires autonomous navigation, a path planner will generate the waypoints and the trajectories that will navigate the MAV to the desired positions. A path planner should guide a MAV through a harsh environment in a mine and guarantee collision avoidance between the agents and the environment.

\section{Path Planner}

The path planner's objective is to generate velocity commands in order to avoid collision, while moving towards the destination. A block diagram of the proposed path planner and the corresponding controller is shown in Figure $\square$. The path planner will generate a velocity $\mathbf{v}_{\mathbf{i}, \mathbf{P F}}$ for the MAV. Then the resulting velocities are converted into position-velocity-yaw trajectories, which can be directly provided to the high level trajectory tracking controller.

A Potential Field Controller (PFC) is implemented for the case of a MAV to avoid any obstacles in its path. $P_{s}=\left(x_{i, s}, y_{i, s}, z_{i, s}\right)$ and $P_{d}=\left(x_{i, d}, y_{i, d}, z_{i, d}\right)$ are 


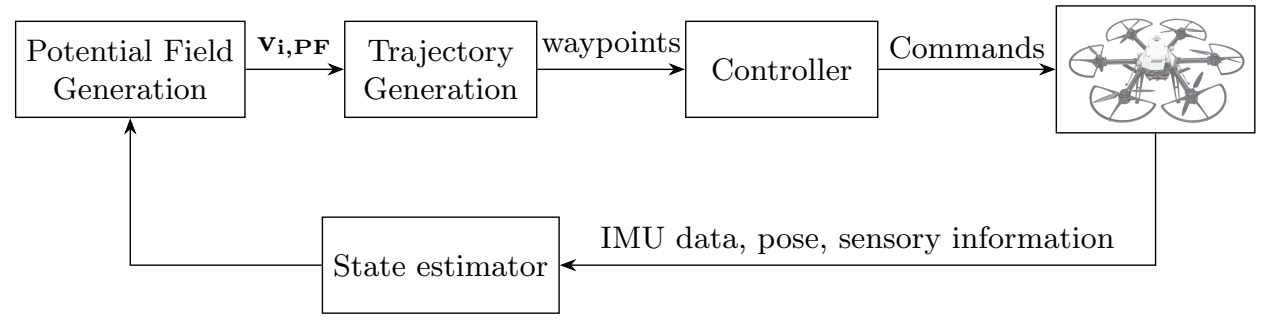

Fig. 2: Proposed method structure.

the current position and the destination position of the $i^{\text {th }}$ agent. The relative distance vector between the agent and the destination is:

$$
P_{s d}=\left[x_{i, s d}, y_{i, s d}, z_{i, s d}\right]^{T}=\left[x_{i, s}, y_{i, s}, z_{i, s}\right]^{T}-\left[x_{i, d}, y_{i, d}, z_{i, d}\right]^{T}
$$

The potential force is defined in $x, y$ and $z$ spatial dimensions and presented as follow:

$$
U_{i, a t t}\left(P_{s}, P_{d}\right)=\frac{1}{2} \beta\left\|P_{s d}\right\|^{2},
$$

where $\beta$ is a positive scale factor and $\left\|P_{i, s d}\right\|$ is the magnitude of the relative distance between the current position of the agent and the destination. The direction and magnitude of the desired movement is computed by calculating the negative gradient of the potential field as follows:

$$
\mathbf{v}_{\mathbf{i}, \text { att }}\left(P_{s}, P_{d}\right)=-\nabla U_{i, a t t}\left(P_{s}, P_{d}\right)=-\beta\left(P_{s}-P_{d}\right)
$$

where $v_{i, a t t}$ is the desired velocity due to the attractive potential field. The repulsive potential is proportional to the inverse square of the distance between the agent and the obstacle $\left(P_{o}\right)$ and is obtained from following equation:

$$
U_{i, r e p}\left(P_{s}, P_{o}\right)=\frac{1}{2} \eta \frac{1}{\left\|P_{s o}\right\|^{2}}
$$

where $\eta$ is a positive scalar factor and $\left\|P_{s o}\right\|$ is the magnitude of the relative distance between the agent and the obstacle. The desired repulsive velocity is obtained as it follows:

$$
\mathbf{v}_{\mathbf{i}, \mathbf{r e p}}\left(P_{s}, P_{o}\right)=-\nabla U_{i, r e p}\left(P_{s}, P_{o}\right)=\eta \frac{P_{s o}}{\left\|P_{s o}\right\|^{4}}
$$

The repulsive force should be zero when the agent has enough safety distance to the obstacle thus:

$$
\mathbf{v}_{\mathbf{i}, \mathbf{r e p}}\left(P_{s}, P_{o}\right)= \begin{cases}\eta \frac{P_{s o}}{\left\|P_{s o}\right\|^{4}} & \text { if }\|d\|<d_{S} \\ 0 & \text { else }\end{cases}
$$


where $d$ is the distance between the agent and the obstacle and $d_{S}$ is the safety distance. A potential field controller is the sum of the attraction and repulsive velocities and can be obtained as it follows:

$$
\mathbf{v}_{\mathbf{i}, \mathbf{P F}}\left(P_{s}, P_{d}, P_{o}\right)= \begin{cases}-\beta\left(P_{s}-P_{d}\right)+\sum_{j=0}^{n_{o}} \eta \frac{P_{j, s o}}{\left\|P_{j, s o}\right\|^{4}} & \text { if }\|d\|<d_{S} \\ -\beta\left(P_{s}-P_{d}\right) & \text { else }\end{cases}
$$

where $n_{o}$ is the number of obstacles. It should be noted that the inspected structure and the position of other agents can be considered as obstacles in the potential force. Moreover, the $v_{i, P F}\left(p_{s}, p_{d}, P_{o}\right)$ should be bounded based on the dynamics of the vehicle.

$$
\mathbf{v}_{\mathbf{i}, \mathbf{P F}}\left(P_{s}, P_{d}, P_{o}\right)^{\text {min }}<\mathbf{v}_{\mathbf{i}, \mathbf{P F}}\left(P_{s}, P_{d}, P_{o}\right)<\mathbf{v}_{\mathbf{i}, \mathbf{P F}}\left(P_{s}, P_{d}, P_{o}\right)^{\max }
$$

\section{Simulations}

This section describes the implementation details of the proposed method as well as simulation results to demonstrate the applicability of the method for underground surveying processes.

\subsection{Implementation Details}

The proposed method has been evaluated in the simulation environment Gazebo along with the Robot Operating System (ROS) framework, where the developed components in the presented simulation results are directly applicable to their real world counterparts. The platform considered in the presented simulations is the Ascending Technologies Firefly hexacopter. Additionally, a minimal sonarbased sensor array, including 8 sonar sensors, is considered in this setup as the main feedback sensor. The ultrasonic array sensor is used for controlling the range measurements from the surrounding obstacles.

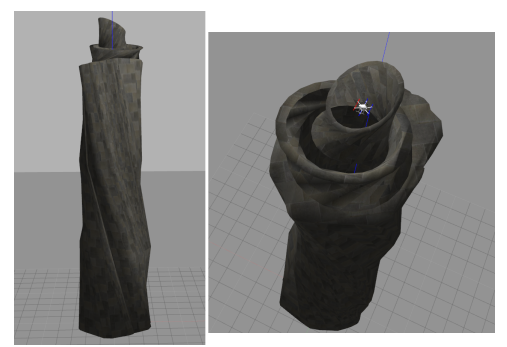

Fig. 3: Simulated shaft in Gazebo framework. On the left outside view, on the right inside view with MAV (Video link [[]]) 


\subsection{Results}

The proposed obstacle-free navigation scheme has been applied in a specific test case, which considers a harsh mine area (vertical shaft). The evaluation of the method is based on the attributes of the obstacle avoidance and mission accomplishment. The mission is considered successful when the aerial platform reaches the goal waypoint. The potential field algorithm considered $\beta=400$ and $\eta=1000$. The safety distance threshold $d_{S}$ to activate the repulsive force of the potential field was set to $3 \mathrm{~m}$. The MAV trajectory is generated based on the attractive part of the potential field converting the forces into position-velocityyaw references. The moment that the repulsive part is activated the positionvelocity-yaw references consider the sum of attractive and repulsive trajectory contributions.
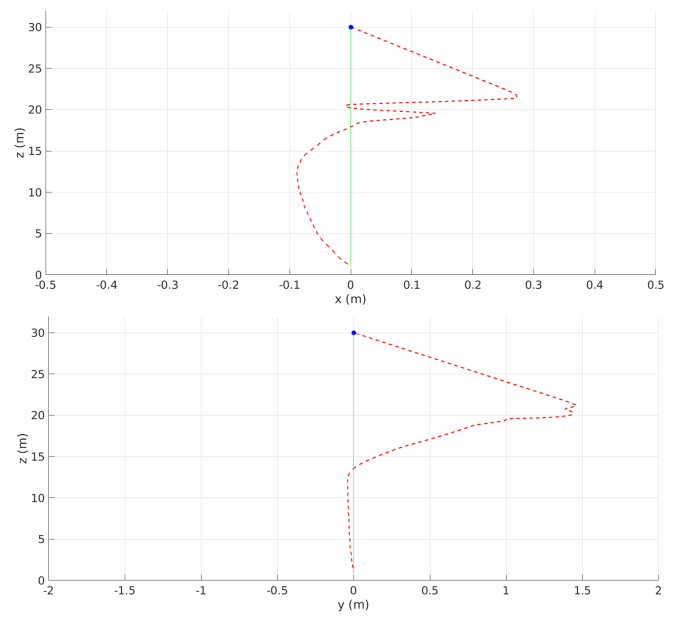

Fig. 4: On top the XZ view of the reference trajectory (green) and actual trajectory (red) followed during the shaft navigation task, on bottom the YZ view of the reference trajectory (green) and actual trajectory (red) followed during the shaft navigation task

Vertical Shaft: In this scenario the MAV is commanded to navigate across the $z$-axis inside the simulated shaft (Figure 3 ). The 3D model of the shaft has an irregular cylindrical shape that eventually collides with the initially planned trajectory for the aerial vehicle, which requires the activation of the proposed navigation scheme. More specifically, the MAV starting from the position $[0 \mathrm{~m}$, $0 \mathrm{~m}, 1.0 \mathrm{~m}]$ is commanded to reach the goal waypoint at $[0 \mathrm{~m}, 0 \mathrm{~m}, 30 \mathrm{~m}]$. The initial designed trajectory is a straight line that connects the starting and final position, depicted in green line in Figure 4 . However, due to the shaft shape some parts of the wall collide with the desired trajectory. The aim of the proposed 
method is to locally re-plan the route that the MAV follows in such a way as to avoid wall collisions and reach the final waypoint, an essential capability for MAV integration in the mining processes.

The resulting trajectory followed is depicted in Figure $\mathbf{\theta}$ (red dashed line), which shows that the MAV was able to reach the goal waypoint, avoiding successfully the obstacle walls along the initial trajectory. During the simulation, the maximum divergence on the $y$-axis was $1.46 \mathrm{~m}$, while the maximum divergence on the $x$-axis was $0.272 \mathrm{~m}$.

\section{Discussion \& Conclusions}

This paper presented the potential application scenarios that make this technology meaningful to employ MAVs underground, while additionally it proposed an efficient sense-and-avoid method for robust navigation in challenging environments. The navigation capabilities consist the backbone of safe and efficient incorporation of MAVs to maintain the integrity of infrastructure assets underground. The performance of the method is highlighted in the presented simulation.

\section{References}

1. K. Schmid, P. Lutz, T. Tomić, E. Mair, and H. Hirschmüller, "Autonomous visionbased micro air vehicle for indoor and outdoor navigation," Journal of Field Robotics, vol. 31, no. 4, pp. 537-570, 2014.

2. P. Gohl, M. Burri, S. Omari, J. Rehder, J. Nikolic, M. Achtelik, and R. Siegwart, "Towards autonomous mine inspection," in Applied Robotics for the Power Industry (CARPI), 2014 3rd International Conference on, pp. 1-6, IEEE, 2014.

3. T. Özaslan, G. Loianno, J. Keller, C. J. Taylor, V. Kumar, J. M. Wozencraft, and T. Hood, "Autonomous navigation and mapping for inspection of penstocks and tunnels with mavs," IEEE Robotics and Automation Letters, vol. 2, no. 3, pp. 17401747, 2017.

4. S. S. Mansouri, C. Kanellakis, E. Fresk, D. Kominiak, and G. Nikolakopoulos, "Cooperative uavs as a tool for aerial inspection of the aging infrastructure," in Field and Service Robotics, pp. 177-189, Springer, 2018.

5. G. Nikolakopoulos, T. Gustafsson, P. Martinsson, and U. Andersson, "A vision of zero entry production areas in minesthis work has been partially funded by the sustainable mining and innovation for the future research program.," 2015. 4th IFAC Workshop on Mining, Mineral and Metal Processing MMM 2015.

6. S. S. Mansouri, P. Karvelis, G. Georgoulas, and G. Nikolakopoulos, "Remaining useful battery life prediction for UAVs based on machine learning," in 20th World Congress of the International Federation of Automatic Control (IFAC), 2017.

7. "MAV shaft navigation simulation video." Available at https://drive.google.com/ open?id=1htDwR0GIF'vgR9vtbW2Q9oajMwKIvgM3h. 